\section{BMJ Open} Paediatrics

\title{
Appendectomy versus non-operative treatment for acute uncomplicated appendicitis in children: study protocol for a multicentre, open-label, non- inferiority, randomised controlled trial
}

Nigel J Hall, ${ }^{1}$ Simon Eaton, ${ }^{2}$ Olivier Abbo, ${ }^{3}$ Alexis P Arnaud, ${ }^{4}$ Marianne Beaudin, ${ }^{5}$ Mary Brindle, ${ }^{6}$ Andreana Bütter, ${ }^{7}$ Dafydd Davies, ${ }^{8}$ Tim Jancelewicz, ${ }^{9}$ Kathy Johnson, ${ }^{10}$ Richard Keijzer, ${ }^{11}$ Eveline Lapidus-Krol, ${ }^{12}$ Martin Offringa, ${ }^{13}$ Nelson Piché, ${ }^{5}$ Risto Rintala, ${ }^{14}$ Erik Skarsgard, ${ }^{15}$ Jan F Svensson, ${ }^{16}$ Wendy J Ungar, ${ }^{13}$ Tomas Wester, $^{16}$ Andrew R Willan, ${ }^{17}$ Augusto Zani, ${ }^{12}$ Shawn D St Peter, ${ }^{10}$ Agostino Pierro ${ }^{12}$

To cite: Hall NJ, Eaton S, Abbo 0, et al. Appendectomy versus non-operative treatment for acute uncomplicated appendicitis in children: study protocol for a multicentre, open-label, non-inferiority, randomised controlled trial. BMJ Paediatrics Open 2017;1:e000028. doi:10.1136/ bmjpo-2017-000028

\section{- Prepublication history for} this paper is available online. To view these files please visit the journal online (http://dx.doi.org/ 10.1136/bmjpo-2017-000028).

Received 3 April 2017 Revised 26 April 2017 Accepted 27 April 2017

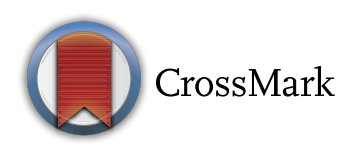

For numbered affiliations see end of article.

Correspondence to Nigel J Hall; n.j.hall@soton. ac.uk

\section{ABSTRACT}

Background Appendectomy is considered the gold standard treatment for acute appendicitis. Recently the need for surgery has been challenged in both adults and children. In children there is growing clinician, patient and parental interest in non-operative treatment of acute appendicitis with antibiotics as opposed to surgery. To date no multicentre randomised controlled trials that are appropriately powered to determine efficacy of nonoperative treatment (antibiotics) for acute appendicitis in children compared with surgery (appendectomy) have been performed.

Methods Multicentre, international, randomised controlled trial with a non-inferiority design. Children (age 5-16 years) with a clinical and/or radiological diagnosis of acute uncomplicated appendicitis will be randomised (1:1 ratio) to receive either laparoscopic appendectomy or treatment with intravenous (minimum 12 hours) followed by oral antibiotics (total course 10 days). Allocation to groups will be stratified by gender, duration of symptoms ( $>$ or $<48$ hours) and centre. Children in both treatment groups will follow a standardised treatment pathway. Primary outcome is treatment failure defined as additional intervention related to appendicitis requiring general anaesthesia within 1 year of randomisation (including recurrent appendicitis) or negative appendectomy. Important secondary outcomes will be reported and a costeffectiveness analysis will be performed. The primary outcome will be analysed on a non-inferiority basis using a $20 \%$ noninferiority margin. Planned sample size is 978 children.

Discussion The APPY trial will be the first multicentre randomised trial comparing non-operative treatment with appendectomy for acute uncomplicated appendicitis in children. The results of this trial have the potential to revolutionise the treatment of this common gastrointestinal emergency. The randomised design will limit the effect of bias on outcomes seen in other studies.

Trial registration number clinicaltrials.gov: NCT02687464. Registered on Jan 13th 2016.

\section{What is already known on this topic?}

- Appendectomy has been the mainstay of treatment of acute appendicitis for over 100 years.

- Recently the need for surgery for uncomplicated acute appendicitis has been challenged and current data suggest the majority of children can be treated with a non-operative treatment pathway instead of surgery.

- The comparative safety and efficacy of non-operative treatment compared with surgery have not yet been determined.

\section{What this study hopes to add?}

This study will determine the relative efficacy of nonoperative treatment compared with appendectomy.

- The randomised study design will help to eliminate bias between treatment groups that may exist in other study types.

- The pragmatic trial design will help to ensure generalisability of trial results.

\section{BACKGROUND}

Acute appendicitis is the most common surgical emergency in children. ${ }^{1}$ The lifetime risk of developing appendicitis is $7 \%-8 \%$, with a peak incidence in the teenage years. The associated financial burden of treating appendicitis is very large.

For over 100 years, surgical removal of the appendix has been deemed necessary to effectively treat acute appendicitis. Appendectomy remains the cornerstone of treatment for acute appendicitis, with the 


\section{Strengths and limitations}

- Large well-designed randomised controlled trial comparing nonoperative management with surgery for children with acute uncomplicated appendicitis.

- Pragmatic diagnostic and treatment protocols.

An international multicentre study.

- Non-blinded.

- May include children without appendicitis since the diagnosis reflects current practice of clinical +/- radiological assessment.

exception of a phlegmon or appendix mass. ${ }^{2}$ However, in recent years this surgical dogma has been challenged and there is a growing literature to suggest that antibiotics without surgery may be an effective treatment for acute appendicitis in adults and more recently in children. This non-operative management of acute appendicitis remains controversial and unproven due to the lack of well designed large prospective randomised controlled trials (RCTs). ${ }^{3}$

Although appendectomy is generally a simple procedure, it requires general anaesthesia and is an abdominal operation with inherent risks and potential complications. Complications related to surgery or anaesthesia occur in over $10 \%$ of children within 30 days of appendectomy. ${ }^{4}$ Although a non-operative approach may avoid these risks and reduce the complication rate, this would not be a viable alternative to surgery unless it is effective at curing acute appendicitis. Another important consideration is that some patients with a clinical and/or radiological diagnosis of acute appendicitis may not actually have acute appendicitis. Even with current imaging methods, $6.3 \%$ of children in Canada and $4.3 \%$ in the
USA undergoing appendectomy are subsequently found to have a normal appendix. ${ }^{5}$ Consequently, this could be considered to be an unnecessary operation. Surgery causes trauma, physiological stress and physical scarring in the child and psychological stress and distress in their parents. A non-operative approach might reduce these. There may be social and economic benefits to the child and family arising from reduced time away from normal daily activities including schooling and parental time off work, and there may be benefits for the healthcare system and society. However, there is the issue of recurrent appendicitis. Following successful non-operative treatment, children would be left with an appendix and be at risk of recurrent appendicitis. The benefits of successful non-operative treatment would only be realised if the rate of recurrent appendicitis is low. If a high proportion of children will develop a recurrence, then there is likely to be less benefit from an initial non-operative approach.

The existing literature relating to the efficacy of non-operative treatment of acute appendicitis is predominantly from adult patients. Several trials and systematic reviews have been reported. ${ }^{36-12}$ In a 2012 meta-analysis Mason et al concluded that while there were benefits to non-operative treatment including fewer complications, better pain control and shorter sick leave, the combined failure and recurrence rates in non-operative patients made this approach less effective overall. ${ }^{11}$ However, in the same year Varadhan et al concluded from their meta-analysis that 'antibiotics can be used safely as primary treatment in patients presenting with acute uncomplicated appendicitis' since $63 \%$ of patients respond to non-operative treatment. $^{12}$

\begin{tabular}{|c|c|c|c|c|c|}
\hline Study & $\begin{array}{l}\text { Country of } \\
\text { origin }\end{array}$ & $\begin{array}{l}\text { Year of } \\
\text { publication }\end{array}$ & Study design & $\begin{array}{l}\text { No. of children receiving } \\
\text { non-operative treatment }\end{array}$ & $\begin{array}{l}\text { Comparative } \\
\text { study* }\end{array}$ \\
\hline Kaneko et $a l^{33}$ & Japan & 2004 & Prospective cohort & 22 & No \\
\hline Abes et $\left.a\right|^{34}$ & Turkey & 2007 & Retrospective cohort & 16 & No \\
\hline Armstrong et $\mathrm{a}^{35}$ & Canada & 2014 & $\begin{array}{l}\text { Non-randomised retrospective } \\
\text { cohort }\end{array}$ & 12 & Yes \\
\hline Koike et $\left.a\right|^{36}$ & Japan & 2014 & Retrospective cohort & 130 & No \\
\hline Gorter et $\mathrm{al}^{27}$ & Holland & 2015 & $\begin{array}{l}\text { Non-randomised prospective } \\
\text { cohort }\end{array}$ & 25 & Yes \\
\hline Hartwich et al ${ }^{37}$ & USA & 2015 & $\begin{array}{l}\text { Prospective parent preference- } \\
\text { based feasibility trial }\end{array}$ & 24 & Yes \\
\hline Minneci et $\left.a\right|^{32}$ & USA & 2015 & $\begin{array}{l}\text { Prospective parent preference- } \\
\text { based trial }\end{array}$ & 37 & Yes \\
\hline Svensson et $a l^{16}$ & Sweden & 2015 & Pilot RCT & 24 & Yes \\
\hline Steiner et $a l^{38}$ & Israel & 2015 & $\begin{array}{l}\text { Non-randomised prospective } \\
\text { cohort }\end{array}$ & 45 & No \\
\hline Tanaka et $\left.a\right|^{39}$ & Japan & 2015 & $\begin{array}{l}\text { Non-randomised prospective } \\
\text { cohort }\end{array}$ & 78 & Yes \\
\hline
\end{tabular}

*Included a comparison group who underwent appendectomy.

$\mathrm{RCT}$,randomised controlled trial. 
In children, the literature is limited (table 1). While antibiotic therapy appears successful in the majority of children with acute uncomplicated appendicitis, no large randomised study of acute appendicitis in children has yet been performed (although there have been RCTs of antibiotic treatment of perforated appendicitis in children ${ }^{1314}$ ). A recent systematic review and meta-analysis of the efficacy of non-operative treatment of acute uncomplicated appendicitis in children demonstrated that non-operative treatment is effective as initial treatment in $97 \%$ of cases. ${ }^{15}$

In preparation for this multicentre RCT, some of our group have performed a pilot RCT at one of the participating centres (Karolinska Institutet, Stockholm). ${ }^{16}$ We have successfully demonstrated feasibility of recruitment to an RCT and demonstrated safety of non-operative treatment of children with acute appendicitis. Furthermore, we have generated pilot data on which our current study is now based. In the pilot RCT all 26 children randomised to appendectomy had histopathologically confirmed acute appendicitis and recovered without significant complications. Only 2 of 24 children in the antibiotic group required appendectomy for histologically proven acute appendicitis within 1 year. Of eligible participants, the recruitment rate was $40 \%$, the dropout rate following treatment allocation was $2 \%$ (1 patient) and no patient was lost to follow-up by 1 year.

Based on these observations, and in response to parents who are now asking whether their child with acute appendicitis really needs an operation, we will perform a large, prospective, multicentre, RCT comparing appendectomy with non-operative treatment in children with acute appendicitis. Our principal research question is: Can children with acute uncomplicated (non-perforated) appendicitis be treated without appendectomy?

\section{METHODS/DESIGN}

\section{Trial design}

The APPY trial has been designed as a pragmatic, parallel-group, unmasked, non-inferiority, multicentre, international, RCT. The protocol has been developed in accordance with the SPIRIT (Standard Protocol Items: Recommendations for Interventional Trials) guideline ${ }^{17}$ and the trial will be conducted and reported according to the CONSORT statement. ${ }^{1819}$ The trial is registered with clinicaltrials.gov: NCT02687464.

\section{Participants}

Children (5-16 years of age) with suspected acute uncomplicated appendicitis will be enrolled. All children with suspected acute non-perforated appendicitis will be assessed by the on-call surgeon who will determine eligibility for the study. This will be based on a clinical and/or ultrasound (US) or CT diagnosis of acute non-perforated appendicitis. The parent(s) and child will be informed of the trial and invited to participate.

\section{Inclusion criteria}

children (age $5-16$ years)

- clinical and/or radiological diagnosis (US and/or CT scan) of acute non-perforated appendicitis

- written informed parental consent in accordance with local regulations and institutional policy

- written informed child assent in accordance with local regulations and institutional policy

\section{Exclusion criteria}

- suspicion of perforated appendicitis

- presentation with an appendix mass or phlegmon (on physical examination and/or imaging)

- non-operative management (two or more doses of intravenous antibiotic) initiated at an outside institution

- previous episode of appendicitis or appendix mass/ phlegmon treated non-operatively

- current treatment for malignancy

- positive pregnancy test

- diagnosis of cystic fibrosis

\section{Randomisation}

After signed informed consent, a standardised data set will be collected from all participants at all participating institutions. Patients enrolled in the study will be randomised to groups (1:1 ratio) using an online stratified randomisation tool, allowing instant assignment to treatment group 24 hours per day with concealment of allocation. Allocation to groups will be stratified taking into account factors that may affect outcome of treatment: (1) Gender: Male; Female; (2) Duration of symptoms $<48$ hours; $>48$ hours; and (3) Centre. Due to the nature of the interventions blinding will not be possible, and as imaging is not an inclusion criterion, it is not possible to stratify by presence/absence of faecolith.

\section{Interventions}

Patients will be allocated to non-operative antibiotic treatment or appendectomy. Figure 1 illustrates patient flow through the two treatment pathways during the acute admission following randomisation.

\section{Non-operative treatment group}

Participants allocated to non-operative treatment will be treated according to a treatment pathway standardised across all centres comprising intravenous fluid treatment, a minimum of 12 hours of intravenous antibiotics, a minimum period of 12 hours taking clear fluid only and regular clinical review. This review is conducted to detect symptoms and signs of clinical deterioration including, but not limited to, increased fever, increased tachycardia, and increased pain or tenderness. An additional formal review will be performed the following day and children who are stable or clinically improving will continue with non-operative treatment.

Children in whom non-operative treatment is successful will receive a minimum of 12 hours intravenous antibiotics 


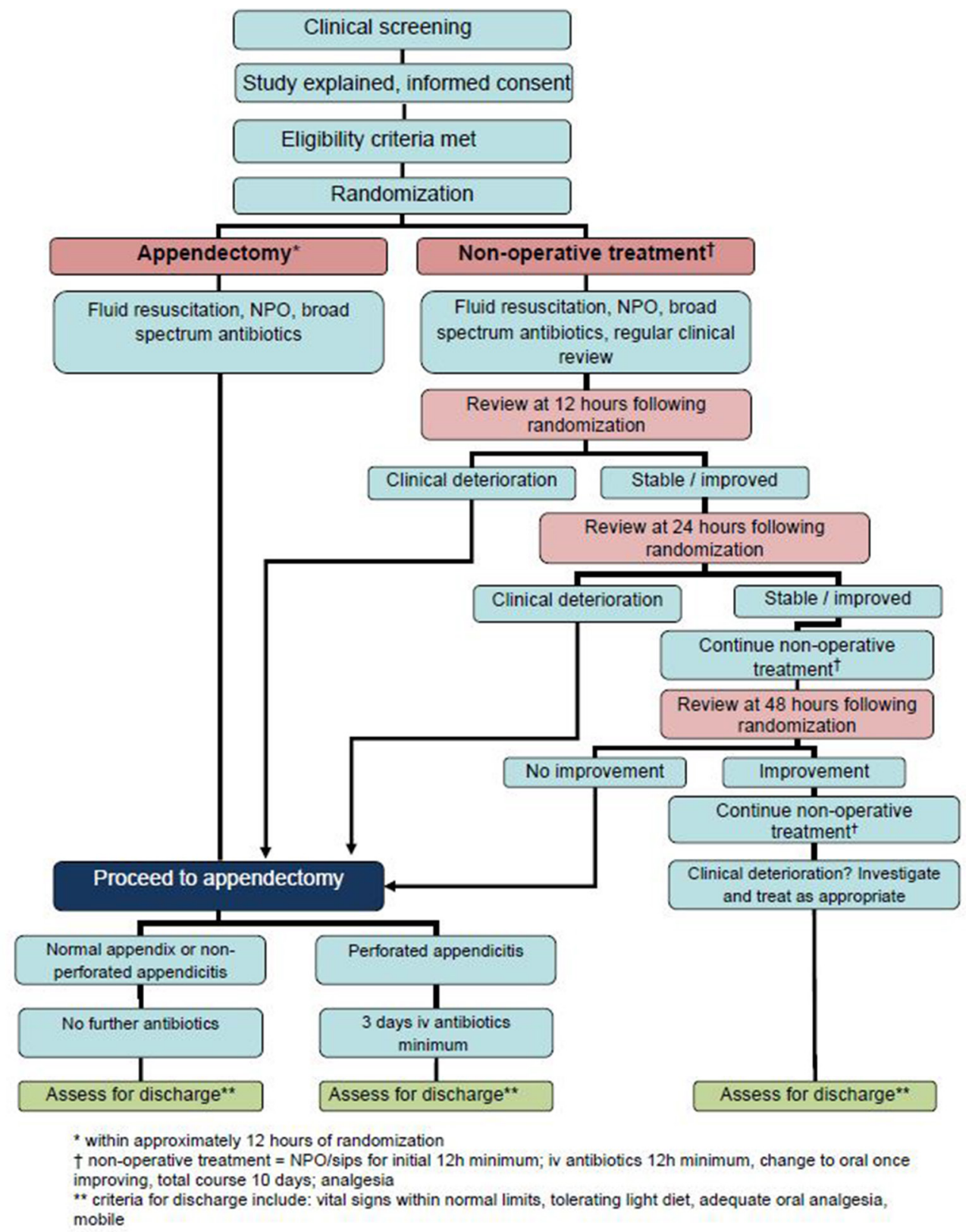

Figure 1 Clinical flow chart for APPY trial.

and then be switched to oral antibiotics once they have shown clinical improvement. They will be discharged home once they meet a standardised set of criteria to be used in all centres: vital signs (including temperature) within normal limits, tolerating a light diet orally, adequate oral pain relief and mobile. They will receive a total course of 10 days of antibiotics (intravenous and oral) following randomisation.

Children within the non-operative treatment group will remain under the direct care of an attending paediatric surgeon. If a child's clinical condition deteriorates at any time, they will undergo laparoscopic appendectomy, and will receive postoperative care identical to that of children in the appendectomy treatment group (see below), and any other care that might be dictated by sound clinical judgement.
The diagnosis of acute appendicitis may be confirmed, or strongly suspected in an otherwise eligible patient at an outside institution, prior to referral to the treating/ trial centre. A widely accepted standard of practice made in consultation with the treating centre, is to administer a single dose of intravenous antibiotic in such patients prior to transfer. These patients will be considered eligible for randomisation provided they have not received more than a single dose of pretransfer intravenous antibiotic. A patient who has received two or more doses of antibiotic prior to evaluation at the treating/trial centre will be considered to have 'commenced conservative treatment', and would therefore be ineligible for randomisation. The choice of antibiotics will vary between centres and will be the antibiotic regimen that is current standard of care in that centre. This is due to (1) varying antibiotic regimes 
among participating centres at present influenced by local factors, including antimicrobial stewardship and drug $\operatorname{cost}^{20}$ and (2) a lack of evidence to support a specific antibiotic regimen for childhood appendicitis. Allowing each centre to maintain current antibiotic protocols will improve study feasibility and increase generalisability of the results. However, the duration of combined intravenous and oral therapy will be standardised to 10 days.

Following discharge, children who receive non-operative treatment will not be offered elective appendectomy. They will be counselled about the risk of recurrence as part of the consent process for the trial using best available data including that arising from our pilot study. Recurrence of appendicitis within the 1-year follow-up period will be treated with appendectomy; these children will not be eligible for re-enrolment.

\section{Appendectomy group}

Children allocated to appendectomy will undergo laparoscopic appendectomy within approximately 18 hours of randomisation which is the current standard of care in all centres participating in this study. Participants will receive intravenous antibiotics from the time of randomisation and be treated postoperatively with intravenous antibiotics according to a defined and standardised treatment regime based on consensus for this trial. Specifically, children with a macroscopically normal appendix or non-perforated acute appendicitis will receive no further antibiotics; children with perforated appendicitis will continue to receive intravenous antibiotics for a minimum of 3 days, and may receive additional antibiotics per local practice. The type of antibiotics used in each centre will be identical to those used in the non-operative treatment group. Following cessation of intravenous antibiotics, criteria for discharge home will be identical to those in the non-operative treatment group.

\section{Outcomes}

Primary outcome

The primary outcome is treatment failure defined as: (1) additional intervention related to appendicitis requiring general anaesthesia within 1 year of randomisation (this includes the recurrence of appendicitis after non-operative treatment, which will be treated with appendectomy) or (2) negative appendectomy. This definition of the primary outcome will capture all important parameters in both treatment groups including specifically: failure of antibiotic treatment requiring appendectomy, significant complication (defined as requiring general anaesthesia) in either treatment group, recurrence of acute appendicitis (treated by appendectomy) and negative appendectomy.

\section{Secondary outcomes}

Secondary outcomes are objective measures of treatment efficacy that fulfil important core areas of relevance to clinicians and patients (pathophysiological manifestations, life impact, resource use and death) ${ }^{21}$ We have selected secondary outcomes which we believe to be important and relevant for future treatment decisions. They will be recorded as they illustrate clinical course and are objectively measurable in a large multicentre RCT:

- complications: adverse events related to either nonoperative treatment of appendicitis or appendectomy which require additional interventions without general anaesthesia, during the first year following randomisation will be categorised according to the Clavien-Dindo classification ${ }^{22}$

- time to discharge home after randomisation measured in hours as a continuous variable

- number and duration of hospital admissions related to appendicitis, appendectomy or their complications during the first year following randomisation

Other secondary outcomes will be collected and compared between treatment groups. We will also undertake a full cost-effectiveness analysis to examine the incremental cost (savings) of non-operative treatment versus appendectomy per treatment failure averted.

\section{Follow-up}

All participants will be seen in the outpatient clinic at 6 weeks following discharge and again at 3 months and 1 year following randomisation for collection of secondary outcome data. Details of any unscheduled healthcare visits specific to the previous episode of appendicitis will be recorded contemporaneously if at the same institution, or will be inquired about at the 3-month and 1-year follow-up appointments. If families are unable to attend for follow-up then consultation by telemedicine facility or telephone will be undertaken.

We will obtain permission from these families to hold their personal contact details in a secure registry and to contact them in the future to determine in a longer follow-up study if they have had complications that may be attributed to treatment (including recurrence of appendicitis)

\section{Sample size calculation}

The sample size has been calculated to test our null hypothesis that non-operative treatment with antibiotics is inferior to appendectomy. Data contributing to our calculations arise from our pilot RCT data, ${ }^{16}$ the existing literature in adult patients and recent (2012) outcomes data from the 14 participating centres.

In the appendectomy treatment group, the estimate of participants meeting criteria for the primary end point is based on the negative appendectomy rate and postoperative need for re-intervention rate, which were estimated from the recent experience collected from each participating centre. We found a $5 \%$ negative appendectomy rate and $2 \%$ postappendectomy rate of intervention requiring general anaesthesia. The anticipated proportion of participants with treatment failure in the appendectomy group is therefore $7 \%$. 
In the non-operative treatment group the estimate of participants meeting criteria for the primary end point is based on a $20 \%$ incidence of additional intervention requiring general anaesthesia related to appendicitis (combination of treatment failure, complication requiring general anaesthesia or recurrent appendicitis)

We will set a non-inferiority margin of $20 \%$ for this study. Thus the primary null hypothesis for this trial is $H_{0}: \mu_{\text {non-op }}-\mu_{o p}>0.2$ (inferiority), where $\mu_{\text {non-op }}$ and $\mu_{o p}$ are the probabilities of the primary outcome occurring in the non-operative arm and the appendectomy arm, respectively. The alternative hypothesis on which the sample size is based on is $H_{1}: \mu_{\text {non-op }}-\mu_{o p} \leq 0.13$ (ie, 20\%-7\%). The power for this trial will be set to $90 \%$; therefore to have a 90\% probability of rejecting $H_{0}$ when $H_{1}$ is true, using a one-sided, 0.05 level test, we will require a total of 880 children (two equal groups of 440). To allow for a combined $10 \%$ drop out and loss to follow-up, we intend to recruit 978 (ie, 880/0.9) children in total. Based on the characteristics of participating centres we estimate recruitment will take place over a period of 24-30 months.

\section{Analysis}

Final analysis will be performed after the final patient has completed 1 year follow-up after randomization. Baseline variables will be compared between groups using the appropriate descriptive statistics. The primary outcome will be analysed by testing, at the $5 \%$ level (one-sided), the null hypotheses $H_{0}: \mu_{\text {non-op }}-\mu_{o p}>0.2$ (inferiority) versus the alternative hypothesis $H_{A 1}: \mu_{\text {non-op }}-\mu_{o p} \leq 0.13$ (non-inferiority), where $\mu_{n o n-o p}$ and $\mu_{o p}$ are the probabilities of the primary outcome occurring in the non-operative arm and the operative arm, respectively. To facilitate this test of hypothesis, the $90 \%$ CI for $\mu_{\text {non-op }}-\mu_{o p}$ will be constructed. If the upper-bound of the CI is less than 0.2 , the null hypothesis will be rejected and the non-operative arm will be declared non-inferior. Time to discharge will be compared between treatment arms using a MannWhitney U-test to account for right skewing from most patients spending a short time in the hospital with few and widely variable protracted stays. The incidence of complications will be compared between treatment arms using a two-sided Fisher exact test. The number of hospital admissions will be compared between treatment arms using a Poisson model and the total duration of hospital admissions in the first year following randomization will be compared between treatment arms using a Mann-Whitney U-test. All outcomes will be analysed on an intention-to-treat basis. We will also analyse outcomes by the stratification criteria (gender, duration of symptoms, centre). As an exploratory analysis in the subset of patients for whom the presence/absence of faecolith is known, an analysis of the primary outcome similar to the one described above will be performed with the presence/absence of appendicolith as a covariate.

To ascertain the efficacy of treatment in the non-operative treatment groups, we will perform an interim analysis for the first half of the planned sample size.
It will not be possible to use the primary outcome as defined for this interim analysis as data contributing to the primary outcome will not become measurable until 1 year following randomisation. With a planned 1 year recruitment period to recruit $\sim 50 \%$ of patients and with a 1 year follow-up, the time point at which this interim analysis would be performed would unavoidably occur near the end of our planned recruitment period (ie, 24 months). We will therefore perform an interim analysis based on a modified primary outcome with a shorter (3 month following randomization) follow-up period. This analysis will be based on all elements of the primary outcome but with shorter follow-up. At the interim analysis we will test at the 0.01 level (one-sided) the hypothesis $H I_{0}: \mu_{n o n-o p}-\mu_{o p} \leq 0.13$ (non-inferiority) versus the alternative hypothesis $H I_{1}$ : $\mu_{\text {non-op }}-\mu_{o p}>0.13$ (inferiority), where $\mu_{\text {non-op }}$ and $\mu_{\text {op }}$ are the probabilities of the 3 month primary end point occurring in the non-operative arm and the operative arm, respectively. If the hypothesis $H I_{0}$ is rejected in favour of $H I_{1}$, patient recruitment will be stopped and the non-operative arm declared inferior. No adjustment for the final analysis will be required since the interim and final analyses test different hypotheses. The interim analysis will be performed blind to the investigators to minimise the effect of bias influencing subsequent patient treatment.

The objective of the economic evaluation is to measure the incremental costs of non-operative management versus surgical treatment for acute non-perforated appendicitis per treatment failure averted from societal and healthcare system perspectives. The design will be a cost-effectiveness analysis that weighs the direct and indirect healthcare costs in both treatment arms against the primary measure of effectiveness-treatment failures. The study will capture all costs and health consequences over a 1-year period following randomisation. Variables listed as secondary outcomes in the proposal (frequency and duration of hospital admissions, surgical interventions, treatment for adverse events and complications) will be included as cost items in the analysis. These analyses will be country-specific to reflect pricing differences. Only direct and indirect costs and resource use that can be attributed to the management of acute appendicitis and related complications will be included. Costs will be aggregated into major categories (intervention, direct healthcare, direct and indirect patient costs), and the mean cost per child will be calculated for each treatment group.

The effect of uncertainty will be tested through extensive sensitivity analysis. Uncertain parameters may include the rate of treatment failures, hospital admission length of stay and cost, and the unit price for costly procedures. The probabilistic sensitivity analysis will also be used to undertake a net monetary benefit calculation. 
Trial oversight and safety monitoring

A Trial Steering Committee (TSC) will be convened to ensure that the trial is conducted to rigorous scientific, clinical and ethical standards. A Data Safety and Monitoring Committee (DSMC) will be convened that is independent of both the trial management group and those providing therapy. Terms of reference and a charter will be developed, based on the DAMOCLES (DAta Monitoring Committees: Lessons, Ethics, Statistics) Study Group $^{23}$ and StaR Child health Standard for Research with Children, ${ }^{24}{ }^{25}$ and agreed at an initial meeting at the beginning of the trial prior to the onset of recruitment. Adverse events will be continuously monitored within each centre and reported to the trial coordinating centre. If any serious or unexpected adverse event occurs it will be reported to the chair of the DSMC chair within 72 hours. A summary of adverse events will be reviewed at interim analysis and the DSMC will make a recommendation to the TSC regarding continuation of the trial on safety grounds.

\section{Ethical considerations}

This study will be conducted in accordance with the principles of the Declaration of Helsinki and 'good clinical practice' guidelines as defined by each trial site. Written informed consent will be obtained from all participants prior to randomisation. Our pilot RCT and ongoing observational cohort studies suggest that non-operative treatment of uncomplicated appendicitis in children is safe. $^{162627}$

\section{DISCUSSION}

The APPY trial is based on the hypothesis that a high proportion of children with acute uncomplicated appendicitis can be successfully treated with broad-spectrum antibiotics thereby avoiding a large number of appendectomies. Previous studies of the use of antibiotics in both adults and children suggest that this is likely to be achievable. Whether non-operative treatment with antibiotics is as effective a treatment as appendectomy for this patient population, however, is a more complex question. We believe this is determined by other factors in addition to the success of the initial treatment. For this reason these parameters are included in our composite primary outcome and include incidence of complications in each group, incidence of negative appendectomy and recurrence of appendicitis.

The selection of an appropriate and relevant primary outcome is important for any RCT. Selection of a primary end point which does not reflect the interests of the stakeholder groups involved in treatment selection for a given pathology is likely to lessen the relevance and impact of a trial. For this reason it has been proposed that core outcome sets (COS) be developed. A COS is an established set of outcomes to be measured when evaluating treatment efficacy for a given condition and is usually arrived at by consensus among multiple stakeholder groups (eg, clinicians, researchers, patients/parents, treatment commissioners). The adoption of a COS will likely ensure that outcomes reported are relevant and of importance to multiple stakeholder groups. Further, use of a COS will ensure that a standardised set of outcome measures is reported as a minimum for a given pathology thereby minimising the heterogeneity in outcome reporting between studies. This will improve comparability between studies in quantitative data synthesis such as meta-analysis. Although efforts are underway to develop a COS for children with acute appendicitis, ${ }^{28}$ a COS does not exist at present.

We have therefore selected a primary end point that we believe reflects the important aspects of treatment outcomes on which we as clinicians and researchers would base future treatment decisions for children with acute appendicitis. We have also been influenced by our discussions with our patients and their parents. Negative appendectomy is a frequent finding in most series of paediatric appendectomy and suggests that an unnecessary operation has been performed. A benefit of a non-operative approach would be to avoid an unnecessary operation, although at the cost of an unnecessary course of antibiotics. Complications of treatment are important when evaluating treatment efficacy. Our definition of complications has been designed to capture the failure of non-operative treatment as well as complications requiring general anaesthesia in either treatment group. General anaesthesia has been selected as a marker of the impact of the complication on the patient as per the widely used Clavien-Dindo classification of surgical complications. ${ }^{22}$ Finally we have included recurrent appendicitis in our primary outcome. If the rate of recurrent appendicitis is high then the benefit of initial non-operative treatment is less. If an appendectomy is going to be required for recurrence then it may as well be performed at first presentation. As the primary motivation of non-operative treatment is to avoid an operation and general anaesthesia, we felt the components of the primary outcome should reflect this motivation and therefore be centred around general anaesthesia. Other complications not requiring a general anaesthetic are extremely important to capture and are therefore included as a specific secondary outcome measure (ie, complications not resulting in general anaesthesia classified according to the Clavien-Dindo Scale). In addition the resource utilisation aspect of these complications will be captured in the economic analysis.

Currently diagnosis of acute appendicitis in participating centres results in a false positive rate of $4 \%-6 \%$, that is, a $4 \%-6 \%$ rate of negative appendectomy. Thus, some of those recovering from non-operative management of suspected acute appendicitis will likely be those false positives who did not have acute appendicitis in the first place, in addition to children with antibiotic-responsive acute appendicitis. As no pathological specimen is taken from those who recover effectively with non-operative treatment, we will not accurately know how many 
of these patients actually had appendicitis. It would not be ethically acceptable to undertake additional tests (eg, CT, laparoscopic biopsy) in order to determine whether these patients actually had appendicitis or not, but we believe that not operating on patients who do not have acute appendicitis is one of the potential benefits of non-operative management.

An additional challenge is how to define efficacy in an RCT such as this. We have selected a non-inferiority design since we wish to evaluate whether non-operative treatment is as effective, but not necessarily more effective, than the current standard of care (appendectomy). If non-operative treatment is as effective as appendectomy, the potential benefits include avoiding surgery and its inherent risks, avoiding general anaesthesia, a potential shorter recovery time, and reduced costs to the institution and the healthcare system. Similar trials in adults have used comparative designs. To determine the efficacy of non-operative treatment we will compare how inferior it is to appendectomy. The non-inferiority margin we are willing to accept will in part determine its efficacy.

There is no accepted guidance regarding the magnitude of a non-inferiority margin for surgical trials. A previous similar study in adults ${ }^{8}$ comparing surgery with non-operative treatment for acute appendicitis in adults used a non-inferiority margin of $10 \%$, which has been criticised by some as being too narrow. ${ }^{29}$ A Cochrane review of appendectomy versus antibiotic treatment for acute appendicitis ${ }^{3}$ proposed a non-inferiority margin of $20 \%$ on the basis that non-operative treatment may be marginally less effective but be more patient friendly, thereby justifying a wider non-inferiority margin. We believe that avoidance of an abdominal operation and general anaesthesia provides enough benefit to the patient to justify this wider non-inferiority margin of $20 \%$. A recently reported RCT in adults used a $24 \%$ non-inferiority margin. ${ }^{9}$ It was felt by the trial investigators that setting a non-inferiority margin of more than $20 \%$ would be too wide, as negative appendectomy is included in the appendectomy group so that a wider margin would be too 'generous' to the non-operative group. In addition, even if the treatment failure rate of non-operative treatment falls outside the non-inferiority limits, the trial will usefully inform the discussion between surgeons, patients and their parents, and non-operative treatment might still be regarded as a viable treatment option, although with a lower success rate.

In the protocol, each centre is allowed to maintain current antibiotic protocols. This is in keeping with current concepts of local antibiotic stewardship and the fact that no single antibiotic regime for acute appendicitis is of proven efficacy over another. It is not the aim of the study to determine an optimal antibiotic therapy for acute appendicitis, but to effectively answer the question 'Is non-operative management of acute appendicitis in children, using current local antibiotic policies, non-inferior to operative management'. It is possible that some individual regimens may be more effective than others, and data from the trial might be used as hypothesis-generating in order to design future studies to optimise antibiotic therapy. However, the trial is not powered to examine differences in antibiotic regimens and as a result of this, comparison of antibiotic regimens is not listed as a specific secondary end point. All centres will use a broad-spectrum approach to overcome the limitations of a narrow-spectrum antibiotic regime encountered by others ${ }^{8} 29$

We have specifically designed the APPY trial as an RCT. We believe the RCT design is the most appropriate methodology to determine the comparative effectiveness of non-operative treatment compared with appendectomy. ${ }^{30}$ We are aware of the use of a 'parent/patient choice' design used by other studies in both adults ${ }^{31}$ and children. ${ }^{32}$ Although parental choice may ultimately prove to be important in the treatment of acute appendicitis in children, we believe this parental choice must be informed by high quality evidence of the treatment failure rates of each approach in identical groups of patients. A parent preference design runs the risk of introducing bias between the treatment arms, indeed such a bias is almost implicit in the act of choice itself. Despite the challenges and limitations of an RCT, we therefore strongly believe that a randomised study introduces less bias and is superior to a parent preference-based study. The $40 \%$ recruitment rate from the pilot RCT suggests that many children and parents are uncomfortable with the possibility of not having the appendix removed, and while we believe that even if the current large-scale trial indicates non-inferiority of non-operative management, there will likely always be some children and their parents who will opt to have an operation. The recruitment rate from the current trial will also be an important metric to gauge potential generalisability on a wider scale.

An additional challenge for many surgical trials in particular is to ensure generalisability of trial findings after completion. This trial will therefore be a pragmatic trial in which we will aim to use existing treatment pathways in use at participating centres yet with adequate standardisation across treatment groups to allow meaningful comparison. Our entry criteria will therefore be based on a clinical and/or radiological diagnosis of acute, uncomplicated (non-perforated) appendicitis. There will be no strict requirement for the diagnosis to be based on US or CT scan. The patients who will be eligible for recruitment to this trial are the very ones who are currently being treated with appendectomy for acute appendicitis no matter how the diagnosis is currently made. Children with a faecolith on imaging or raised white cell count or C-reactive protein will all be eligible for inclusion. Finally the laparoscopic approach will be the standard for children in the appendectomy arm since this is the approach in current standard use at participating institutions.

Author affiliations

${ }^{1}$ University Surgery Unit, Faculty of Medicine, University of Southampton, Southampton, Hampshire, UK 
${ }^{2}$ Developmental Biology \& Cancer Programme, UCL Great Ormond Street Institute of Child Health, London, UK

${ }^{3}$ Pediatric Surgery Department, Hôpital des Enfants, Centre Hospitalier Universitaire Toulouse, Toulouse, France

${ }^{4}$ Paediatric Surgery Department, Hôpital Sud, Centre Hospitalier Universitaire, Rennes, France

${ }^{5}$ Division of PediatricSurgery, Centre Hospitalier Universitaire Sainte-Justine, Montreal, Canada

${ }^{6}$ Departments of Surgery and Community Health Sciences, Alberta Children's Hospital, Calgary, Alberta, Canada

${ }^{7}$ Division of Pediatric Surgery, Children's Hospital,London Health Sciences Centre, London, Ontario, Canada

${ }^{8}$ Department of General and Thoracic Surgery, IWK Health Centre, Halifax, Nova Scotia, Canada

${ }^{9}$ Division of Pediatric Surgery, Le Bonheur Children's Hospital, University of Tennessee Health Science Center, Memphis, Tennessee, USA

${ }^{10}$ Department of Surgery, Children's Mercy Hospitals and Clinics, Kansas City, Missouri, USA

${ }^{11}$ Department of Surgery, University of Manitoba, Winnipeg, Manitoba, Canada ${ }^{12}$ Division of Thoracic and General Surgery, The Hospital for Sick Children, Toronto, Ontario, Canada

${ }^{13}$ Child Health Evaluative Sciences, SickKids Research Institute, Toronto, Ontario, Canada

${ }^{14}$ Department of Pediatric Surgery, Children's Hospital, Helsinki University Central Hospital, University of Helsinki, Helsinki, Finland

${ }^{15}$ Department of Surgery, BC Children's Hospital, Vancouver, British Columbia, Canada

${ }^{16}$ Department of Pediatric Surgery, Astrid Lindgren Children's Hospital, Karolinska University Hospital, Stockholm, Sweden

${ }^{17}$ Ontario Child Health Support Unit, SickKids Research Institute, Toronto, Ontario, Canada

Contributors All authors have contributed to study design and writing of the protocol. This manuscript was drafted by NJH with help from SE. The draft was revised by ES, SDSP, AP, WJU and ARW. All authors have seen and approved the final version of the manuscript.

Competing interests None declared.

Ethics approval The study has received ethical approval in the following centres: Children's Mercy Hospital, Kansas City, Missouri, USA; BC Children's Hospital, Vancouver, British Columbia, Canada; Children's Hospital of Western Ontario, London, Ontario, Canada; Children's Hospital Winnipeg, Winnipeg, Mannitoba, Canada; Karolinska University Hospital, Stockholm, Sweden; Hospital for Children and Adolescents, University of Helsinki, Finland. The study is undergoing ethical review in the remaining centres.

Provenance and peer review Not commissioned; externally peer reviewed.

Open Access This is an Open Access article distributed in accordance with the Creative Commons Attribution Non Commercial (CC BY-NC 4.0) license, which permits others to distribute, remix, adapt, build upon this work non-commercially, and license their derivative works on different terms, provided the original work is properly cited and the use is non-commercial. See: http://creativecommons.org/ licenses/by-nc/4.0/

(C) Article author(s) (or their employer(s) unless otherwise stated in the text of the article) 2017. All rights reserved. No commercial use is permitted unless otherwise expressly granted.

\section{REFERENCES}

1. St Peter SD, Sharp SW, Holcomb GW, et al. An evidence-based definition for perforated appendicitis derived from a prospective randomized trial. J Pediatr Surg 2008:43:2242-5.

2. Emil S, Laberge JM, Mikhail P, et al. Appendicitis in children: a ten-year update of therapeutic recommendations. J Pediatr Surg 2003;38:236-42.

3. Wilms IM, de Hoog DE, de Visser DC, et al. Appendectomy versus antibiotic treatment for acute appendicitis. Cochrane Database Syst Rev 2011;11:CD008359.

4. Tiboni S, Bhangu A, Hall NJ, et al. Outcome of appendicectomy in children performed in paediatric surgery units compared with general surgery units. Br J Surg 2014;101:707-14.
5. Cheong LH, Emil S. Outcomes of pediatric appendicitis: an international comparison of the United States and Canada. JAMA Surg 2014;149:50-5.

6. Eriksson S, Granström L. Randomized controlled trial of appendicectomy versus antibiotic therapy for acute appendicitis. $\mathrm{Br}$ J Surg 1995;82:166-9.

7. Styrud J, Eriksson S, Nilsson I, et al. Appendectomy versus antibiotic treatment in acute appendicitis. a prospective multicenter randomized controlled trial. World J Surg 2006;30:1033-7.

8. Vons C, Barry C, Maitre S, et al. Amoxicillin plus clavulanic acid versus appendicectomy for treatment of acute uncomplicated appendicitis: an open-label, non-inferiority, randomised controlled trial. Lancet 2011;377:1573-9.

9. Salminen P, Paajanen H, Rautio T, et al. Antibiotic therapy vs appendectomy for treatment of Uncomplicated acute appendicitis: the APPAC Randomized clinical trial. JAMA 2015;313:2340-8.

10. Hansson J, Körner U, Khorram-Manesh A, et al. Randomized clinical trial of antibiotic therapy versus appendicectomy as primary treatment of acute appendicitis in unselected patients. Br J Surg 2009:96:473-81.

11. Mason RJ, Moazzez A, Sohn $\mathrm{H}$, et al. Meta-analysis of randomized trials comparing antibiotic therapy with appendectomy for acute uncomplicated (no abscess or phlegmon) appendicitis. Surg Infect 2012;13:74-84

12. Varadhan KK, Neal KR, Lobo DN. Safety and efficacy of antibiotics compared with appendicectomy for treatment of uncomplicated acute appendicitis: meta-analysis of randomised controlled trials. BMJ 2012;344:e2156.

13. Blakely ML, Williams R, Dassinger MS, et al. Early vs interval appendectomy for children with perforated appendicitis. Arch Surg 2011;146:660-5.

14. St Peter SD, Aguayo P, Fraser JD, et al. Initial laparoscopic appendectomy versus initial nonoperative management and interval appendectomy for perforated appendicitis with abscess: a prospective, randomized trial. J Pediatr Surg 2010;45:236-40.

15. Georgiou R, Eaton S, Stanton MP, et al. Efficacy and safety of Nonoperative treatment for acute appendicitis: a Meta-analysis. Pediatrics 2017; 139:e20163003

16. Svensson JF, Patkova B, Almström M, et al. Nonoperative treatment with antibiotics versus surgery for acute nonperforated appendicitis in children: a pilot randomized controlled trial. Ann Surg 2015;261:67-71.

17. Chan AW, Tetzlaff JM, Altman DG, et al. SPIRIT 2013 statement: defining standard protocol items for clinical trials. Ann Intern Med 2013:158:200-7.

18. Moher D, Hopewell S, Schulz KF, et al. CONSORT 2010 explanation and elaboration: updated guidelines for reporting parallel group randomised trials. BMJ 2010;340:c869.

19. Schulz KF, Altman DG, Moher D, et al. CONSORT 2010 Statement: updated guidelines for reporting parallel group randomised trials. Trials 2010:11:32.

20. Gerber JS, Kronman MP, Ross RK, et al. Identifying targets for antimicrobial stewardship in children's hospitals. Infect Control Hosp Epidemiol 2013;34:1252-8.

21. Boers M, Idzerda L, Kirwan JR, et al. Toward a generalized framework of core measurement areas in clinical trials: a position paper for OMERACT 11. J Rheumatol 2014;41:978-85.

22. Dindo D, Demartines N, Clavien PA. Classification of surgical complications: a new proposal with evaluation in a cohort of 6336 patients and results of a survey. Ann Surg 2004;240:205-13.

23. DAMOCLES Study Group, NHS Health Technology Assessment Programme. A proposed charter for clinical trial data monitoring committees: helping them to do their job well. Lancet 2005;365:711-22.

24. Ellenberg S, Fernandes RM, Saloojee $\mathrm{H}$, et al. Standard 3: data monitoring committees. Pediatrics 2012;129(Suppl 3):S132-S137.

25. Hartling L, Wittmeier KD, Caldwell PH, et al. StaR Child Health: developing evidence-based guidance for the design, conduct, and reporting of pediatric trials. Clin Pharmacol Ther 2011;90:727-31.

26. Minneci PC, Sulkowski JP, Nacion KM, et al. Feasibility of a nonoperative management strategy for uncomplicated acute appendicitis in children. J Am Coll Surg 2014;219:272-9.

27. Gorter RR, van der Lee JH, Cense HA, et al. Initial antibiotic treatment for acute simple appendicitis in children is safe: shortterm results from a multicenter, prospective cohort study. Surgery 2015;157:916-23.

28. Hall NJ, Kapadia MZ, Eaton S, et al. Outcome reporting in randomised controlled trials and meta-analyses of appendicitis treatments in children: a systematic review. Trials 2015;16:275. 
29. Mason RJ. Appendicitis: is surgery the best option? Lancet 2011:377:1545-6.

30. Svensson JF, Patkova B, Almström M, et al. Design of studies for Antibiotic treatment of acute appendicitis in Children: in support of RCTs. Ann Surg 2015:1.

31. Di Saverio S, Sibilio A, Giorgini E, et al. The NOTA Study (Non Operative treatment for acute appendicitis): prospective study on the efficacy and safety of antibiotics (amoxicillin and clavulanic acid) for treating patients with right lower quadrant abdominal pain and longterm follow-up of conservatively treated suspected appendicitis. Ann Surg 2014;260:109-17.

32. Minneci PC, Mahida JB, Lodwick DL, et al. Effectiveness of Patient Choice in Nonoperative vs Surgical Management of Pediatric Uncomplicated acute appendicitis. JAMA Surg 2016;151:1-8

33. Kaneko K, Tsuda M. Ultrasound-based decision making in the treatment of acute appendicitis in children. $J$ Pediatr Surg 2004;39:1316-20.
34. Abeș M, Petik B, Kazil S. Nonoperative treatment of acute appendicitis in children. J Pediatr Surg 2007:42:1439-42.

35. Armstrong J, Merritt $\mathrm{N}$, Jones $\mathrm{S}$, et al. Non-operative management of early, acute appendicitis in children: is it safe and effective? $J$ Pediatr Surg 2014;49:782-5.

36. Koike Y, Uchida K, Matsushita K, et al. Intraluminal appendiceal fluid is a predictive factor for recurrent appendicitis after initial successfu non-operative management of uncomplicated appendicitis in pediatric patients. J Pediatr Surg 2014;49:1116-21.

37. Hartwich J, Luks FI, Watson-Smith D, et al. Nonoperative treatment of acute appendicitis in children: a feasibility study. J Pediatr Surg 2016;51:111-6.

38. Steiner Z, Buklan G, Stackievicz R, et al. A role for conservative antibiotic treatment in early appendicitis in children. $J$ Pediatr Surg 2015;50:1566-8.

39. Tanaka $\mathrm{Y}$, Uchida $\mathrm{H}$, Kawashima $\mathrm{H}$, et al. Long-term outcomes of operative versus nonoperative treatment for uncomplicated appendicitis. J Pediatr Surg 2015;50:1893-7. 